\title{
Estimation of Cardiac Output by Bioimpedance Cardiography
}

\author{
Major M J World \\ BSc, MD, MRCP (UK), RAMC \\ Consultant Physician \\ Cambridge Military Hospital, Aldershot
}

SUMMARY: Changes in thoracic electrical bioimpedance during the cardiac cycle have been related to the ejection of the stroke volume of blood during cardiac systole. Refinements in the recording and analysis of these changes permit estimation of cardiac output. Encouraging reports comparing results obtained by this method and those obtained simultaneously using the current standard invasive method have been published. While there are limitations, the advantages of this technique are sufficient to make bioimpedance cardiography attractive to the military physician. It is for these reasons that the principles of this technology are reviewed in this paper.

\section{Introduction}

There are many reasons for measuring cardiac output as part of a full haemodynamic assessment. One of the most important is in the context of intensive therapy. Several recent studies have indicated that the rate of oxygen delivery to the peripheral tissues is the critical factor which determines survival in patients who are seriously ill. Determination of the total rate of oxygen delivery necessitates estimation of haemoglobin concentration, oxygen saturation of arterial blood and cardiac output. In the seriously ill patient where anaemia and any deficiencies in oxygenation of pulmonary arterial blood have been corrected, it has been suggested that cardiac output should be increased to values higher than normal in order to elevate oxygen delivery to the peripheral tissues and thereby enhance the probability of survival ${ }^{1}$.

In some patients, estimation of total oxygen delivery to the periphery may be insufficient to assess the likelihood of survival. In one prospective study of outcome in patients requiring ventilation for acute respiratory failure and receiving intensive therapy for additional problems ${ }^{2}$, the only feature that distinguished survivors from non-survivors was the change in total oxygen consumption in response to a vasodilator induced increase in cardiac output. In non-survivors, an otherwise covert oxygen debt was revealed when total oxygen delivery was increased in response to prostacyclin-induced vasodilatation. In these circumstances, total oxygen consumption increased only in the non-survivors and this was apparent when the oxygen saturation of mixed venous blood failed to rise in these patients.

Apart from other considerations, cardiac output is the most important indicator of cardiac performance and should be monitored routinely in critically ill patients in order to observe a satisfactory response to manoeuvres intended to increase and maintain cardiac output. Ideally, the method employed to estimate cardiac output should be accurate and give immediate, continuous results. It should also be non-invasive in order to avoid unnecessary hazards thereby permitting use by appropriate hospital staff in addition to

physicians so that the largest number of patients can be monitored. Thoracic electrical bioimpedance (TEB) cardiography satisfies most of these criteria. For this reason, this paper examines this method for estimating cardiac output in some detail.

\section{The Instrument}

The non-invasive continuous cardiac output monitor 3 (revision 7) (NCCOM3-R7) is manufactured by으. BoMed Medical Manufacturing Ltd, Irvine, Californ USA. The current cost is $£ 8900$ plus VAT. It is $\vec{b} \subseteq$ completely portable box measuring $30 \times 29 \times 14 \mathrm{~cm}$ and $\bar{\nabla}$. weighs $3.2 \mathrm{~kg}$. There is a liquid crystal display which shows the values of six variables with each heartbeat for the average obtained over a fixed number of heartbea or over a fixed time interval. It has a key pad to ente essential patient and chronological data and determine the mode of analysis and display. Furthet details and arrangements for demonstration are available from the UK agents: Kimal Scientific Products $\unrhd$ Ltd, Arundel Road, Uxbridge, Middlesex UB8 2SA.

\section{Operation}

For practical convenience, all instructions including application of electrodes and entry of patient and chronological data are displayed on the upper surface of the monitor. Eight good quality silver/silver chloride electrocardiographic (ECG) electrodes of $5 \mathrm{~cm}$ diameter are connected to the colour coded patient lead array of the NCCOM 3 and are then applied to the cleansed skin surface of the patient as shown in Fig. 1.

The upper group of four electrodes is placed as two sets of two electrodes on either side of the root of the neck. The lower group of four electrodes is placed as two

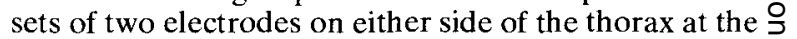
level of the xiphisternum. As shown in Fig 1, the $\frac{7}{2}$ ipsilateral pairs of cervical and thoracic electrodes constitute a functional set. The ipsilateral electrodes $N$ which are further apart (upper cervical and lower thoracic) are used to apply an extremely low magnitude $(2.5 \mathrm{~mA}$ RMS $=$ root mean square $)$, high frequency $(70$ $\mathrm{KHz}$ ) alternating current. The closer pair of ipsilateral 
electrodes (lower cervical and upper thoracic) are employed to detect changes in electrical potential. In addition to voltage changes related to changes in TEB, the same electrodes detect changes in surface potential related to the ECG.

In some patients, paticularly the obese, the ECG (and in particular the R wave of the ECG) cannot be detected without application of an additional pair of electrodes. These are applied to the anterior chest wall at the extremities of the anticipated or determined axis of ventricular depolarization (Fig 1.)

The importance of accurate detection of the ECG is shown in Fig 4 and it is for this reason that failure to detect the ECG will result in failure to display data.

Once all electrodes are applied and connection made to the monitor, which for preference should have been switched on some five minutes earlier, certain data have to be entered via the keypad. These are the sex, height $(\mathrm{cm})$, weight $(\mathrm{kg})$ and a six figure patient identity number which could be the date of birth. Finally, the internal clock is set and once this is completed, the NCCOM3 should start to display cardiac output values with each beat of the heart; so-called "fast" mode. Additional haemodynamic variables derived from the TEB signal are displayed simultaneously. It is possible to index data by body weight or by body surface area.
The values obtained from sixteen acceptable beats car be averaged, so-called "slow" mode.

Whichever display format is chosen, a printer can bez connected to the monitor in order to print the displayed $\mathbb{B}$ data and indicate the time of day that the data were? collected (Fig 2.)

\section{Principles of Thoracic Electrical Bioimpedanc Cardiography}

The idea that the resistance to conduction of electrica? current across the thorax might vary during the cardiag cycle and in some way might reflect cardiac pump performance is an attractive one. Unfortunately, the basal resistance of the thorax to a direct current is high and the variations related to cardiac activity are so smalb that reliable detection and quantification of suchchanges by this means is virtually impossible.

However, by employing a small alternating current electrical differentiation of the detected resultan voltage change with respect to time is possible and woulक enable the equivalent of change of resistance with $\vec{\omega}$ respect to time to be computed. Subsequent integration? could then yield the magnitude of the originaR fluctuations in resistance related to cardiac ang

respiratory activity.
The resistance to an alternating current is a comptex?

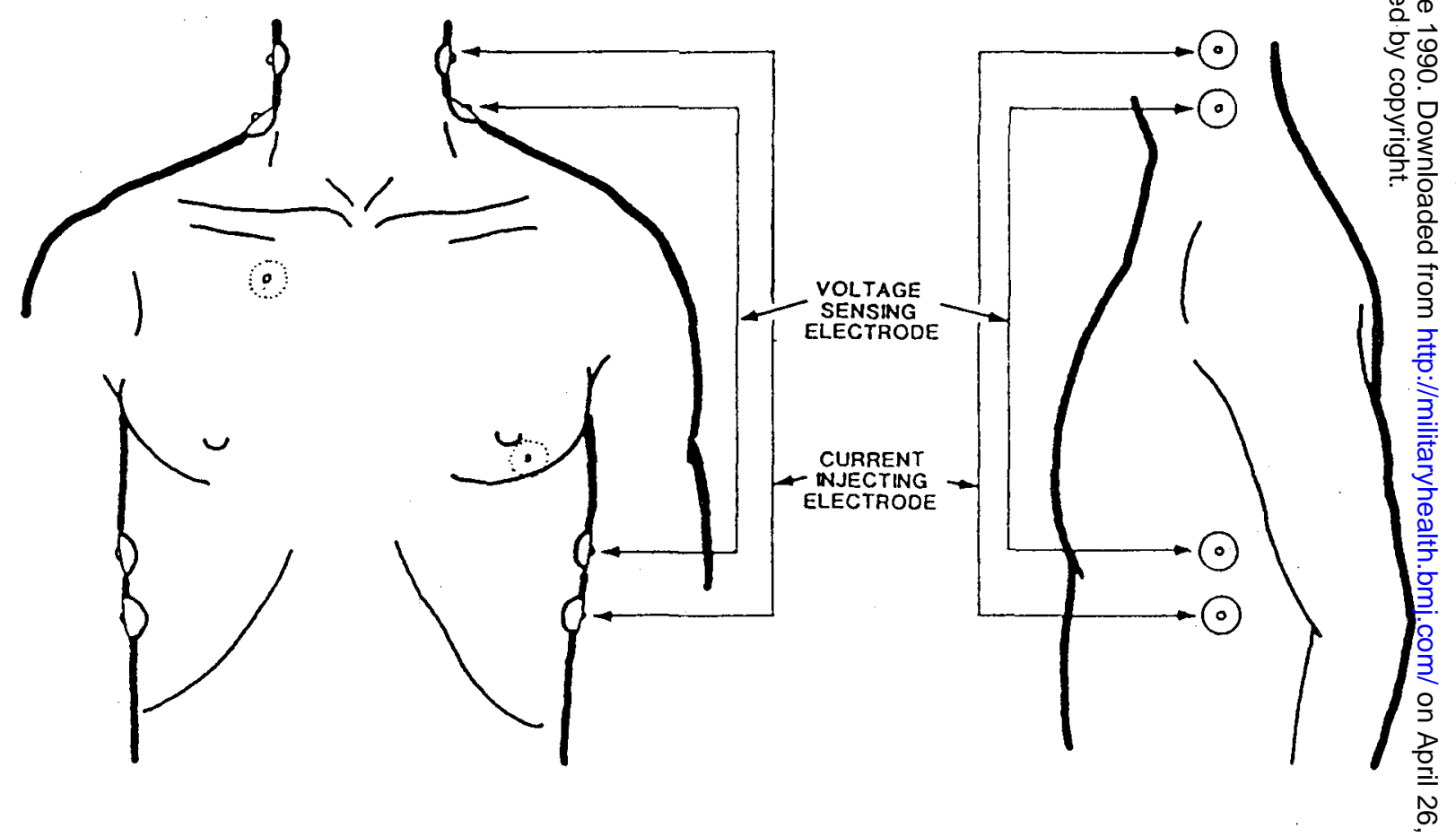

Fig 1. Location of surface electrodes for TEB cardiography by NCCOM3. The centres of each pair of electrodes must be $5 \mathrm{~cm}$ apari Circles with a broken circumference in the right infraclavicular and left inframammary locations are the sites of application of auxillar $\mathrm{W}^{\circ}$ ECG electrodes (see text for further details). 
BoMed NCCOM3-R7

CARDIODYNAMIC MONITOR

$\begin{array}{lr}\text { Patient } & \text { male } \\ \text { ID \# } & 230861 \\ \text { height } & 183 \mathrm{~cm} \\ \text { weight }(0.95) & 077 \mathrm{~kg} \\ \text { VEPT } & 07170 \mathrm{ml} \\ \text { BSA } & 2.00 \mathrm{msq}\end{array}$

CO SV EDV PF EF HR TFI IC ER STR ACI TIME

CO SV EDV PF EF HR TIME

$\begin{array}{llllll}: 06.2 & 108 & 187 & 067 & 57 & 058\end{array}$

$: 06.6 \quad 120 \quad 216 \quad 076 \quad 55 \quad 055$

$: \begin{array}{llllll}05.8 & 112 & 201 & 070 & 55 & 052\end{array}$

$: 06.2 \quad 114 \quad 200 \quad 070 \quad 56 \quad 055$

$: \begin{array}{llllll}06.5 & 114 & 201 & 071 & 56 & 057\end{array}$

$: 06.1 \quad 110 \quad 184 \quad 068 \quad 59 \quad 055$

$: 06.2 \quad 109 \quad 179 \quad 067 \quad 60 \quad 057$

CO SV EDV PF EF HR TFI IC ER STR ACI TIME $\begin{array}{lllllllllll}: 06.5 & 115 & 196 & 072 & 58 & 056 & 23.6 & 050 & 29 & 040 & 138\end{array}$

$: 06.4 \quad 112 \quad 193 \quad 070 \quad 57 \quad 057 \begin{array}{lllllll}23.8 & 049 & 30 & 041 & 136 & 10: 02\end{array}$

$: 05.3 \quad 093 \quad 166 \quad 060 \begin{array}{lllllllll}165 & 056 & 23.8 & 042 & 29 & 043 & 118 & 10: 05\end{array}$

$\begin{array}{lllllllllllll}05.5 & 097 & 181 & 063 & 53 & 056 & 23.6 & 044 & 28 & 047 & 118 & 10: 10\end{array}$

Indices by BSA

CI SI EDI PFI EF HR TFI IC ER STR ACI TIME

$: \begin{array}{lllllllllll}02.7 & 045 & 089 & 306 & 50 & 059 & 23.6 & 042 & 29 & 051 & 117\end{array}$

$\begin{array}{llllllllllll}02.7 & 045 & 085 & 302 & 52 & 060 & 23.4 & 042 & 30 & 049 & 113 & 10.11\end{array}$

Indices by weight

CI SI EDI PFI EF HR TFI IC ER STR ACI TIME

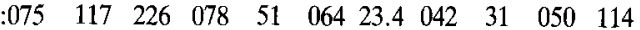

$\begin{array}{llllllllllll}: 073 & 115 & 222 & 077 & 51 & 064 & 23.4 & 041 & 31 & 050 & 115\end{array}$

Fig 2. Examples of the different forms of print-out possible having entered the sex, height, weight and six figure identity number (e.g. date of birth) for the patient and set the internal clock. Requesting results after every heartbeat limits the printing to values for the first six variables alone. Having calculated mean values after 16 heartbeats permits values for all twelve variables to be printed.

\begin{tabular}{|c|c|c|c|}
\hline ACI & -Acceleration index & HR & - Heart rate \\
\hline BSA & - Body Surface Area & IC & - Index of contractility \\
\hline $\operatorname{Co}(\mathbf{1})$ & - Cardiac Output (Index) & PF(I) & -Peak Flow (Index) \\
\hline & $\begin{array}{l}\text {-End Diastolic Volume } \\
\text { (Index) }\end{array}$ & SV(I) & $\begin{array}{l}\text {-Stroke Volume } \\
\text { (Index) }\end{array}$ \\
\hline $\mathbf{E F}$ & - Ejection Fraction & TFI & - Thoracic Fluid Index \\
\hline
\end{tabular}

ER -Ejection Ratio

variable which depends not only upon the ratio of the amplitudes of voltage to current for a given conductor but also on the frequency of the applied current. This distinction from resistance is reflected in the term impedance (Z). As previously mentioned, NCCOM 3 employs a steady, small magnitude (2.5mA RMS), high frequency $(70 \mathrm{KHz})$ current devoid of adverse biological effect. This is applied across the length of the human thorax resulting in generation of a potential difference (voltage) which can be detected and quantified. This potential difference should be constant if impedance, is constant. As explained above, this is not the case.
Superimposed on basal impedance $\left(\mathrm{Z}_{\mathrm{o}}\right)$ are fluctuations in impedance (AZ) related to the respiratory and cardiac cycle, the latter being by far the most numerous over a given time interval. Electronic differentiation of impedance with respect to time yields $\mathrm{dZ} / \mathrm{dt}$ and eliminates the basal impedance detected previously. This technique greatly diminishes variations in impedance related to respiration but does not eliminate them. Subsequent integration of $\mathrm{dZ} / \mathrm{dt}$ also permits calculation of the magnitude of the original fluctuation in impedance (AZ). When recordings are made in voluntary apnoea or, more conveniently, the fluctuations related to respiration are identified electrically, averaged and subtracted, the fluctuations related to the cardiac cycle remain (Fig 3) and it is to these cardiac fluctuations in impedance that the term $\mathrm{AZ}$ will refer in the discussion which follows. As these fluctuations in TEB are related temporarily to the ejection of the stroke volume of blood during cardiac systole and that the waveform is similar to that of systemic arterial pressure (Fig 4) it is believed that it is the pumping activity of the heart that is the cause of these fluctuations. Therefore a theoretical relationship between stroke volume and fluctuation in TEB has been sought.

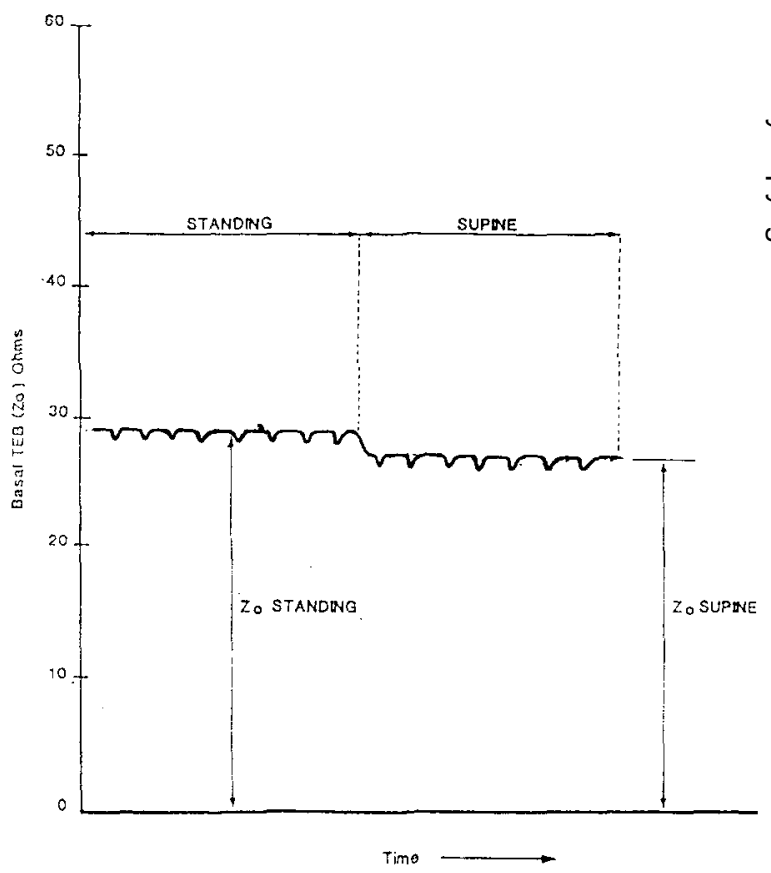

Fig 3. Basal TEB $\left(Z_{0}\right)$ bearing superimposed fluctuations related to the cardiac cycle as seen in voluntary apnoea. Adopting the supine position after standing results in an increased blood content of the thorax with resulting reduced impedance. 
ECG

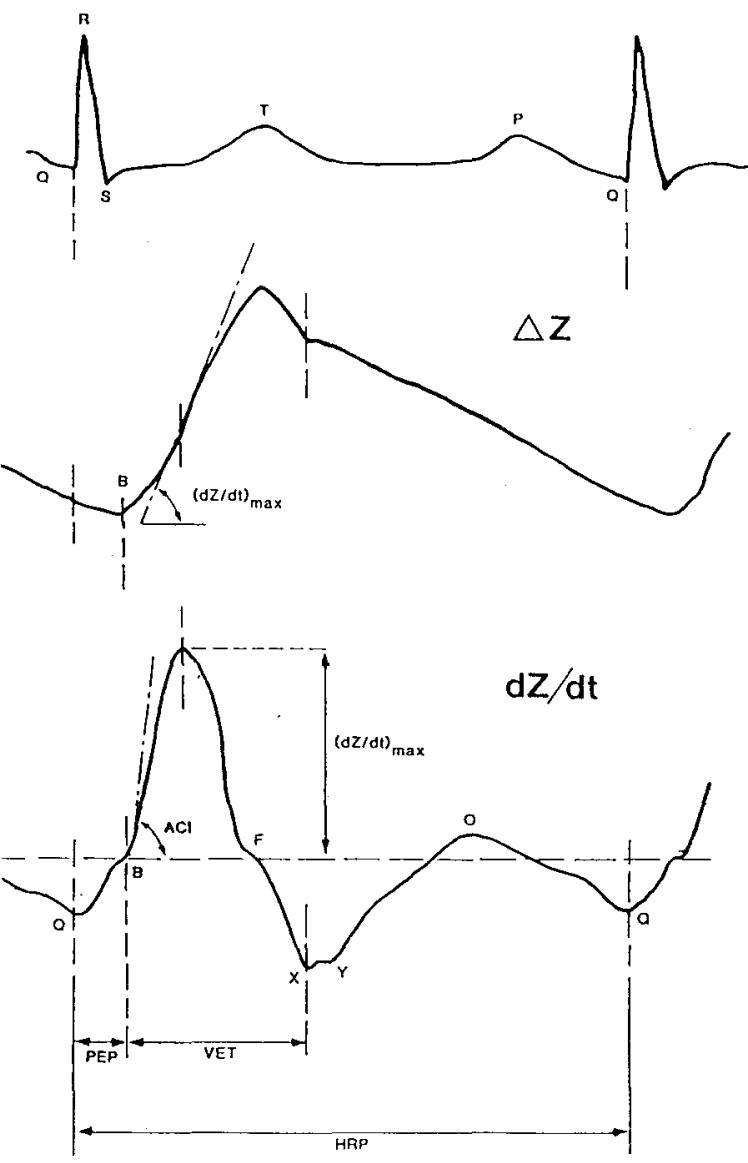

Fig 4. Time relationships of ECG, changes in impedance (AZ) and the rate of change of impedance with respect to time $(\mathrm{dZ} / \mathrm{dt})$.

\begin{tabular}{|c|c|c|c|}
\hline $\begin{array}{l}\mathrm{ACI} \\
\mathrm{B} \\
\mathrm{dZ} / \mathrm{dt}_{\max }\end{array}$ & $\begin{array}{l}\text { - Acceleration Index } \\
\text { - Aortic valve opening } \\
\text { - Ejection Velocity Index }\end{array}$ & $\begin{array}{l}\text { PEP } \\
\text { VET } \\
X \\
Y\end{array}$ & $\begin{array}{l}\text { - Pre-ejection period } \\
\text { - Ventricular ejection time } \\
\text { - Aortic valve closure } \\
\text { - Pulmonary valve closure }\end{array}$ \\
\hline $\begin{array}{l}\text { F } \\
\text { HRP } \\
0\end{array}$ & $\begin{array}{l}\text {-Reflected wave } \\
\text { - Heart rate period } \\
\text {-Mitral valve opening }\end{array}$ & & \\
\hline
\end{tabular}

\section{Theoretical Considerations}

For ease of reference, the derivation of the mathematical relationship between stroke volume and the change in TEB is given in the Appendix to this paper. The essential concept, however, is that with each ejection of the stroke volume of blood into the thoracic aorta, there is a corresponding increase in the volume of this electrical conductor with the consequence that there is a transient reduction in impedance (AZ). Provided allowance is made for the variation in dimension of the human thorax, it is possible to estimate stroke volume and hence cardiac output of a given individual from the change in TEB which occurs with each heartbeat in that? individual.

The NCCOM3 Monitor will display the estimates of the following variables:

Peak Flow

Ejection Fraction

End Diastolic Volume

Cardiac Output

Heart Rate

Stroke Volume

It is possible to display one of a number of additional $\overrightarrow{\vec{F}}$ variables details of which can be obtained from the literature of the manufacturer.

\section{Comparison with other methods}

The clinical methods available to estimate cardiac $\stackrel{\mathbb{\Phi}}{\Omega}$ output have been the subject of a recent authoritative@ review $^{6}$ which described briefly the principles upon $\rightarrow$ which each method was based and commented on ${ }^{\circ}$ reliability. It should be consulted for further details. $\vec{\omega}$ Amongst the methods reviewed, mention was made of $\stackrel{5}{-}$ magnetic resonance imaging and left ventriculography. These methods are not considered here as their nature and cost preclude routine clinical use in the intensive care setting.

In order to determine whether the TEB estimate of cardiac output is clinically useful, the criterion of ${ }^{\prime}$ paramount importance is that there should be good agreement with a standard method. Although nळo absolute standard method for determination of cardiac ${ }^{-}$ output exists, the Fick method does have a position of $ᄃ$ relative pre-eminence. One study employing a whoBy $\widehat{D}$ non-invasive variant of the Fick method to estimas $\overrightarrow{0}$ cardiac output suggested that while the correspondiag $\mathscr{E}$ TEB estimates obtained simultaneously weo significantly correlated, they were inaccurate, being consistently lower ${ }^{7}$. However, data presented in the same paper indicated that the particular version of the Fick method employed was itself inaccurate, tending to $\frac{\mathrm{D}}{\mathbb{Q}}$ over-estimate cardiac output for a given rate of oxygen $\stackrel{\varrho}{\rightarrow}$ consumption when compared with three studies $\overrightarrow{\overrightarrow{0}}$ published previously employing the direct (invasive) Fick method. This observation indicates that further studies comparing TEB and Fick estimates of cardiac output are essential.

Whether or not a good and accurate correlation is ultimately shown with the Fick method under conditions? of rest and exercise, and a report (Sramek personal $\overline{\widetilde{\Phi}}$ communication) suggests that it soon will be, the really important situation to examine is that of the seriously ill: patient in the intensive care unit. In the latter 3 . circumstance, the thermodilution method is used routinely and with confidence to estimate cardiac output. There are several reports ${ }^{8-13}$ which indicate goodo agreement between simultaneous TEB and? thermodilution estimates of cardiac output witho correlation coefficients ranging between 0.71 and 0.95 . In one study where 391 paired estimates were reported ${ }^{9}, \sigma$ over $80 \%$ of values lay within $\pm 20 \%$ variation from the line of identity.

On balance, the results of these comparative studies 
are most encouraging and certainly do not provide overwhelming evidence to reject TEB estimates of cardiac output under all circumstances. The advantages and limitations of this non-invasive technique as embodied in the commercially available NCCOM 3 are indicated in Table 1.

\section{Discussion}

The TEB estimate of cardiac output has been found to be inaccurate in patients with aortic incompetence, marked dysrrhythmia and septicaemia. It is virtually impossible to estimate cardiac output employing TEB during thoracotomy (see Bernstein ${ }^{3}$ for further details). Aortic incompetence should be of little military consequence if enlistment is prevented when potential recruits with this lesion are detected at preliminary medical examination. Myocardial damage frequently results in dysrrhythmia. In younger soldiers, myocarditis is more likely than myocardial infarction to be the cause of this and could affect large numbers of troops during a viral epidemic. Similarly, septicaemia is a condition likely to occur in military patients and is associated with under-estimation of cardiac output. In this situation, the TEB estimate errs on the side of safety unlike dysrrhythmia where overestimation of cardiac output is possible. In all these circumstances, alternative methods must be employed if a more reliable estimation of cardiac output is required.

One particular advantage of TEB is the speed with which estimates of cardiac output can be obtained from a large number of patients. Once TEB electrodes and leads are attached to patients, the monitor can be switched from patient to patient quite quickly. Despite the initial data entry necessary before obtaining a reading from a given patient, it is possible to monitor a much larger number of patients in a given time period than with a thermodilution cardiac output computer. Leaving aside the time and skill required to insert a

Table 1. Comparison of advantages and limitations of the different clinical methods for estimation of cardiac output.

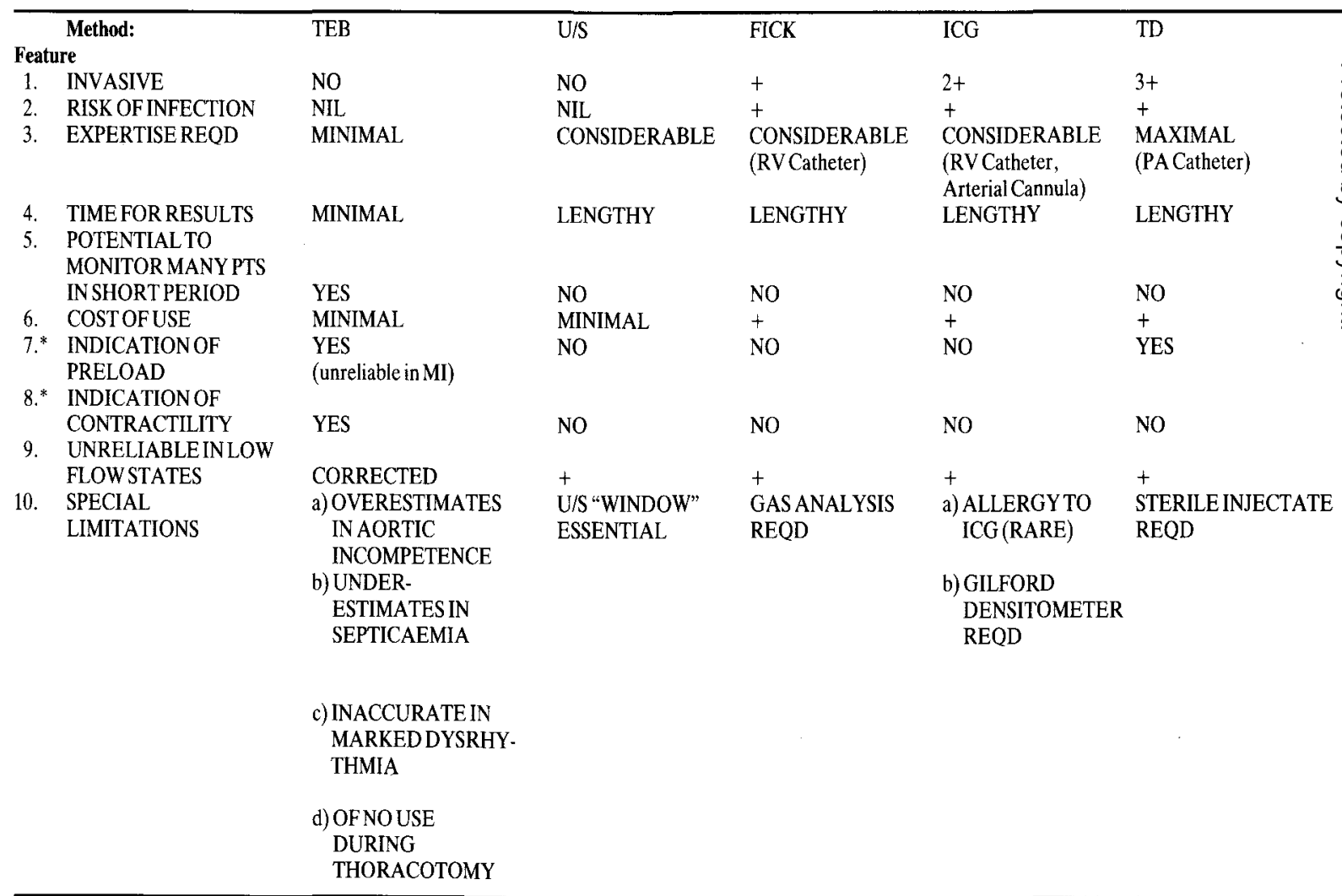


Swan-Ganz catheter, the time necessary to prepare several injectate syringes to obtain one reliable estimate of cardiac output using thermodilution is prohibitive. The fact that the TEB technique is non-invasive permits its use by technicians releasing medical and nursing staff for other essential duties.

Brief mention of the pulse oximeter must be made during this discussion. This instrument reveals the presence of pulsatile blood flow, the pulse rate and oxygen saturation of arterial blood in a continuous, noninvasive, objective fashion. At best it indicates the presence or absence of the minimum conditions to permit life. None of the variables monitored by the pulse oximeter have been shown to distinguish survivors from non-survivors in patients who are seriously ill ${ }^{1}$. The instrument does not indicate precisely the rate of oxygen delivery to the tissues and therefore does not indicate the cardio-pulmonary reserve available to combat additional stress. While the pulse oximeter indicates whether there is any possibility of continued life, it does not indicate the probability.

Under operational conditions, the military physician can expect to contribute to the direction of treatment of large numbers of shocked patients over short periods. Where survival seems likely to be dependent on operative treatment, the goal of resuscitation must be to exceed the same total oxygen delivery threshold as that shown to maximize the chance of survival of civilian patients in similar circumstances. By attending to airway, breathing and circulation, basic skills may be sufficient to restore adequate haemodynamic stability, arterial oxygen saturation and hence consciousness to the majority of injured patients. Where consciousness is not restored and the probability of cerebral trauma or hypoxic damage of an irreversible nature seems remote, more detailed assessment may be necessary. Provided local vascular injury is excluded and oxygenation of pulmonary arterial blood is considered adequate, the failure of a pulse oximeter to detect peripheral pulsatile circulation of blood with satisfactory oxygen saturation will be due to systemic arterial hypotension secondary to low cardiac output. At this point, monitoring by TEB cardiography will be of value to identify the restoration of cardiac output which should occur once transfusion (preferably with blood so that oxygen - carrying capacity is preserved) results in venous return sufficient to generate adequate cardiac filling pressures. High filling pressures (central venous pressure) without improved cardiac output would suggest immediate thoracotomy is desirable to exclude cardiac tamponade if circumstances permit such priority treatment.

Once restored, cardiac output and hence total oxygen delivery, should continue to be monitored both per- and post-operatively. In this way, any deterioration which would otherwise jeopardise chances of survival of the patient would be rapidly detected so that effective remedial action which will abort the development of complications otherwise generated by hypoxia could be를 taken.

TEB cardiography could be the practical solutions which permits an objective basis for certain criticab military clinical decisions. Estimation of cardiac outpue may aid the decision of when and to whom scarces? operating time should be allocated and ensure that, once committed, this precious resource is unlikely to be wasted.

\section{Appendix}

Theoretical Basis for Estimating Stroke Volume from Cardiogenic Change in Thoracic Electrical Bioimpedance

Throughout the discussions which follow, the change infw thoracic electrical bioimpedance $(\Delta \mathrm{Z})$ which accompanies the ejection of stroke volume is considered positive whereas ejection of blood from the heart is, in fact, associated with reduced impedance. A fuller discussion of the theoretical relationship between stroke volume and fluctuations in thoracion electrical bioimpedance can be found elsewhere ${ }^{3}$.

The temporal relationship between the ECG, fluctuation in thoracic electrical bio-impedance $(\Delta \mathrm{Z})$ and its derivative (dZ dt) can be seen in Fig 4 .

Consider a length ( $1 \mathrm{cms}$ ) of descending thoracic aorta cross sectional area $\mathrm{A} \mathrm{cm}^{2}$ and that the specific resistivity blood is ${ }_{b}(\mathrm{Ohm} . \mathrm{cm}$.). Then the impedance $(\mathrm{ZOhms})$ is gi by

$$
z=\rho \frac{l}{b^{A}} \cdots \cdots \cdots(1)
$$

Whilst the length of the thoracic aorta is considered fixed, cross sectional area of the vessel $\left(A_{V}\right)$ increases transiently ejection of the stroke volume so that the volume of the vesseb $\left(V_{v}\right)$ varies with time $(t)$.

$$
A_{v(t)}=\frac{v_{v(t)}}{l} \text { or } v_{v(t)}=A_{v(t)} \cdot l
$$

From Equation (1)

Multiplying both sides by $l$

$$
A_{v(t)}=\rho_{b} \cdot{\frac{l^{2}}{Z_{v(t)}}}
$$

$$
A_{v(t)} \cdot l=\rho_{b} \cdot \frac{l}{z}_{v(t)}^{2}
$$

or

$$
v_{v(t)} \rho_{D} \cdot \frac{l^{2}}{z_{v(t)}} \ldots \ldots \ldots(2)
$$

As the thoracic aorta is encased within the thorax, it is no possible to measure impedance of the aorta directly. If the thorax is considered to be a cylinder (with fixed impedance $\mathrm{Z}_{\mathrm{c}}$ 至. containing the thoracic aortic cylinder (with impedance $\left.Z_{v(t)}\right)$ then with these conductors in parallel, the measurable mutua impedance $\left(Z_{\mathbf{m}(t)}\right)$ is given by

$$
{\frac{1}{Z_{m(t)}}}=\frac{1}{z_{c}}+\frac{1}{z_{v(t)}}
$$

\section{.}


or,

$$
{\frac{1}{z_{v(t)}}}=\frac{1}{\bar{z}_{m(t)}}-\frac{1}{\bar{z}_{c}}
$$$$
=\frac{Z_{c}-Z_{m}(t)}{Z_{m(t)} \cdot Z_{c}}
$$

or,

$$
z_{v(t)}=\frac{z_{m(t)} \cdot Z_{c}}{z_{c}-Z_{m(t)}}
$$

It has been found ${ }^{3}$ that $Z_{m(t)}$ and $Z_{c}$ differ by less than one percent and approximate to the basal TEB, $Z_{0}$.

$$
\text { Hence, } \quad z_{v(t)} \bumpeq \frac{z_{0}^{2}}{\Delta z^{2}(t)}
$$

Then substituting in equation (2)

$$
v_{v(t)} \bumpeq \rho_{b} \cdot \frac{l^{2}}{z_{0}^{2}} \cdot \Delta z_{(t)}
$$

The variation in vascular volume with time is more usually indicated by $\Delta \mathrm{V}_{\mathrm{v}}$.

so,

$$
\Delta v_{v} \bumpeq \rho_{b} \cdot \frac{l^{2}}{z_{0}^{2}} \cdot \Delta z_{(t)}
$$

This equation indicates that if there is a relationship between stroke volume and the change in volume of the thoracic aorta, then stroke volume should be related in turn to the change in TEB. Differentiating variation in TEB $(\Delta Z)$ with respect to time gives $\mathrm{dZ} / \mathrm{dt}$. From equation(3), it can be deduced that if $V_{v}$ and $\Delta Z$ are related, then $d Z / d t$ will be related to $d V_{v} / d t$, ie blood flow.

Similarly $\mathrm{d} Z / \mathrm{dt}_{\max }$ will be related to $\mathrm{dV}_{\mathrm{v}} / \mathrm{dt}_{\max }$ ie peak blood flow. If this peak blood flow was constant throughout the ejection phase of cardiac systole, then stroke volume (SV) would vary thus:

$$
\text { SV } \mathcal{C} d z / d t_{\max } \text {. LVET }
$$

where $\mathrm{LVET}=$ left ventricular ejection time

The product $\left(\mathrm{dZ} / \mathrm{dt}_{\max }\right.$. LVET) indicates the magnitude of the change in TEB $\left(\Delta Z^{\prime}\right)$ at the end of ventricular systole if $\mathrm{dZ} / \mathrm{dt}_{\max }$ was constant throughout the ejection phase.

i.e.

$$
\Delta Z^{\prime}=d Z / d t_{\max } \cdot \text { LVET }
$$

substituting in equation (3)

$$
S V=\rho_{b} \cdot \frac{l^{2}}{z_{0}^{2}} \cdot d z / d t_{\max }
$$

Returning to the thorax, if basal TEB $=Z_{0}$ and the specific resistivity of the thorax $=\rho_{\mathrm{T}}$ then

$$
Z_{0}=\rho_{T} \cdot \frac{l}{A_{T}} \quad \text { where } A_{T}=\text { cross-sectional area of thorax }
$$

so,

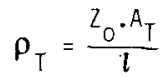

but

$$
A_{T}=\frac{V_{T}}{l} \quad \text { so, } \quad \rho_{T}=\frac{z_{0} \cdot V_{T}}{l^{2}}
$$

Previously, it has been assumed that $\rho_{\mathrm{b}}=\boldsymbol{\rho}_{\mathrm{T}}$. However, more recent work ${ }^{4}$ has shown experimentally that $\rho_{\mathrm{T}}=2 \boldsymbol{P}_{\mathrm{b}}$. For the purposes of discussion, it can be assumed that $\rho_{b}={ }_{T} \rho_{\text {Then, }}$ substituting in equation (4).

and simplifying:

$$
S V=\frac{Z_{0} \cdot V_{T}}{l^{2}} \cdot \frac{l^{2}}{z_{0}^{2}} \cdot d z / d t_{\max } \cdot \text { LVET }
$$

$$
S V \simeq V_{T} \cdot\left(\frac{d Z / d t_{\text {max }}}{Z_{O}}\right)
$$

In the human thorax, it has been found that the circumference $\mathrm{C}$ at the level of xiphoid process is related to the length of the thorax such that:

$$
\text { or } \quad \begin{array}{rlrl}
c & =3 l \\
\text { so } & & 2 \pi r & =3 l \\
& r & =\frac{3 l}{2 \pi}
\end{array}
$$

Hence the cross sectional area at the level of the xiphoid process, $\mathbf{A}_{\mathrm{T}}$ is given by:

$$
\begin{aligned}
A_{\mathrm{T}} & =\pi(3 l / 2 \pi)^{2} \\
& =9 l^{2} / 4 \pi \\
& =l^{2} / 1.4
\end{aligned}
$$

Therefore, if considered cylindrical, the volume of the thorax $\mathrm{V}_{\mathrm{T}}$ is given by

$$
v_{T}=l^{3} / 1.4
$$

Substituting in equation (5)

$$
S V=\frac{l^{3}}{1.4} \cdot \frac{\mathrm{dz} / \mathrm{dt} \mathrm{t}_{\max }}{\mathrm{z}_{0}} .
$$

Experimentally, this equation has been found to overestimate stroke volume by a factor of 3 . There are at least three possible sources for this error. Firstly, the product $\left(\mathrm{dZ} / \mathrm{dt}_{\max }\right.$. LVET) overestimates the impedance image $\left(\Delta Z^{\prime}\right)$ of stroke volume as it assumes that the maximal rate of ejection of blood from the left ventricle $\left(\mathrm{dV} / \mathrm{dt}_{\max }\right)$ is constant throughout the ejection phase of systole. Secondly, it has been mentiond that $\rho_{\mathrm{T}}=2 \boldsymbol{\rho}_{\mathrm{b}}$ and if incorporated in equation (6) this experimental finding would halve the estimated value for SV. Finally, the thoracic volume $V_{\mathrm{T}}$ assumes the volume of the thorax to be a cylinder whereas it is smaller, approximating to a truncated cone.

It is therefore not surprising that empirically, it has been found that:

$$
S V=\frac{b^{3}}{4.2} \cdot\left(\frac{d z / d t_{\max }}{z_{0}}\right) \cdot \text { LVET }
$$

To eliminate errors in measuring thoracic length, 1 , it has been found that $\mathrm{I}$ is a fixed proportion of height $(\mathrm{H} \mathrm{cms})$ such that $I$ $=0.17 \mathrm{H}$. A further correction, $\delta$ to equation (7) to allow for deviation from ideal body weight $(\mathrm{W} \mathrm{kg})$ has been incorporated such that

$$
\delta=\boldsymbol{\beta} \cdot \frac{W(\text { observed })}{W(\text { ideal })}
$$


where $\boldsymbol{\beta}=$

blood vol $(\mathrm{ml} / \mathrm{kg})$ at given $\%$ deviation from ideal body weight body $\mathrm{vol}(\mathrm{ml} / \mathrm{kg})$ at ideal body weight

Thus,

$$
S V=\delta \cdot \frac{(0.17 \mathrm{H})^{3}}{4.2} \cdot\left(\frac{\mathrm{dz} / \mathrm{dt} t_{\max }}{z_{0}}\right) \cdot \text { LVET .........(8) }
$$

This is the Sramek equation ${ }^{5}$. It is incorporated into the NCCOM3 monitor. Once the sex, height and weight of the patient are entered, the values of remaining variables on the right hand side of equestion (8) can be determined as shown in Fig 4 and stroke volume can be calculated.

\section{REFERENCES}

1. Shoemaker W C, Bland R D and Appel P L. Therapy of critically ill postoperative patients based on outcome prediction and prospective clinical trials. Surg Clin North Am 1985; 65: 811-833.

2. BIHARID et al. The effects of vasodilation with prostacyclin on oxygen delivery and uptake in critically ill patients. $N$ Engl J Med 1987; 317: 397-403.

3. BERNSTEIN D P. Noninvasive cardiac output measurement. In: Shoemaker W C, Ayres S, Grenvik A et al (Eds.) Textbook of critical care, Chapter 16 pp 159-185 Philadelphia: W B Saunders Co, 1989.

4. MeIJER J H et al. Differential impedance plethysmography for measuring thoracic impedances. Med Biol Eng Comput 1982; 20: 187-194.
5. Sramek B B, Rose D M and Miyamoto A. Stroke volume equation with a linear base impedance model and its accuracy, as compared to thermodilution and magnetic 3 flowmeter techniques in humans and animals. Proceedings $\stackrel{\mathbb{Q}}{\propto}$ of the Sixth International Conference on Electrical $O$ Bioimpedance, Zadar, Yugoslavia, 1983 p38.

6. EDITORIAL Measurement of cardiac output. Lancet 1988 ; ii: $257-258$.

7. SMith S A et al. Automated non-invasive measurement of cardiac output: comparison of electrical bioimpedance ando carbon dioxide rebreathing techniques. Br Heart J 1988;59: 등 292-298.

8. MAtTAR J A et al. A clinical comparison between cardiac $\frac{\overrightarrow{ }^{\circ}}{2}$ output measured by thermodilution versus non-invasive $\varrho$ thoracic electrical bioimpedance. Acute Care 1986; 12: 5860.

9. APPEL P L et al. Comparison of measurement of cardiac. output by bioimpedance and thermodilution in severely ill surgical patients. Crit Care Med 1986; 14: 933-935.

10. Spinale F, Reines H D and Crawford F A. Electricalbioimpedance as a method for continuous non-invasive 3 estimation of cardiac output: experimental and clinical studies. Crit Care Med 1987; 15: 304.

11. SHELLOCK G and JAKUBOWSKI A. Measurement of cardiac output in critically ill patients by a new non-invasive bioimpedance system: comparison with the thermodilution method. Physiologist 1987; 30: 215.

12. WONG D $\mathrm{H}$ et al. Non-invasive cardiac output: two measurement methods compared with thermodilution a the importance of measured heart rate and ejection tim $c$ Anesth Analg 1988; 67: (Supplement): S262.

13. SALANDIN Vet al. Comparison of cardiac output estimation by thoracic electrical bioimpedance, thermodilution and $\notin$ Fick methods. Crit Care Med 1988; 16: 1157-1158. 